\title{
ChemComm
}

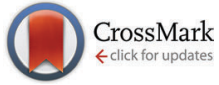

Cite this: Chem. Commun., 2015, 51, 8675

Received 12th March 2015, Accepted 20th April 2015

DOI: $10.1039 / \mathrm{c} 5 \mathrm{cc} 02134 \mathrm{k}$

www.rsc.org/chemcomm

\section{Separation of liquid domains in model membranes induced with high hydrostatic pressure $\dagger$}

\author{
Nicola L. C. McCarthy, Oscar Ces, Robert V. Law, John M. Seddon and \\ Nicholas J. Brooks*
}

\begin{abstract}
We have imaged the formation of membrane microdomains immediately after their induction using a novel technology platform coupling high hydrostatic pressure to fluorescence microscopy. After formation, the ordered domains are small and highly dynamic. This will enhance links between model lipid assemblies and dynamic processes in cellular membranes.
\end{abstract}

Lateral inhomogeneity in biological membranes has been linked with many cellular functions including protein sorting and signal transduction $^{1,2}$ and there is now evidence that this membrane organization must be highly dynamic and take place on a small length scale. ${ }^{3}$ While biological membranes are extremely complex mixtures of thousands of different lipid types and proteins, lateral fluid phase separation has also been observed in highly compositionally simplified model lipid membranes consisting of just three components, although the structures observed in these systems are significantly larger than those thought to exist in cells.

Under suitable conditions, mixtures of a low melting temperature $\left(T_{\mathrm{m}}\right)$ lipid, a high $T_{\mathrm{m}}$ lipid and a sterol or stanol can form bilayers with two coexisting fluid phases; the liquid disordered $\left(\mathrm{L}_{\mathrm{d}}\right)$ phase region, where the lipid hydrocarbon chains exhibit a high degree of conformational disorder, is enriched the low $T_{\mathrm{m}}$ lipid while the liquid ordered $\left(\mathrm{L}_{\mathrm{o}}\right)$ phase, where the hydrocarbon tails exhibit higher conformational order, is enriched in the high $T_{\mathrm{m}}$ lipid and sterol or stanol. The $\mathrm{L}_{\mathrm{o}}$ phase has properties that have been strongly linked to those hypothesized for microdomains in biological membranes. ${ }^{4}$

Whilst the formation of $\mathrm{L}_{\mathrm{o}}$ phases in model membranes has been extensively studied as a function of composition and temperature, ${ }^{5,6}$ potential links to microdomain formation in cells remain uncertain. ${ }^{7}$ It is extremely challenging to create and probe small, dynamic phase separated domains in model systems, but new technologies to measure the structure and

Department of Chemistry, Imperial College London, South Kensington Campus, London SW7 2AZ, UK. E-mail: nicholas.brooks@imperial.ac.uk

$\dagger$ Electronic supplementary information (ESI) available: Materials and methods, further details of high pressure microscopy system and videos showing domain growth in GUVs. See DOI: 10.1039/c5cc02134k dynamics of model membranes very soon after their perturbation offers the prospect of beginning to bridge this gap.

High pressure can be used to drive phase changes in model membrane systems and has significant advantages over the use of temperature changes. Pressure equilibrates across the sample extremely rapidly (at the speed of sound), allowing microdomains to be studied very soon after their induction. In addition, changing pressure at constant temperature allows volume effects to be studied without a change in thermal energy ${ }^{8,9}$ and pressure changes can be applied extremely quickly both up and down. ${ }^{10}$

Pressure will always drive a reduction in overall volume and in lipid assemblies, the hydrocarbon chain region is more laterally compressible than the headgroups..$^{10}$ Because of this, changing pressure can lead to changes in membrane interfacial curvature, ${ }^{11-13}$ however moderate pressure increases can simply cause an increase in the conformational ordering of the lipid hydrocarbon chains, which can lead to $\mathrm{L}_{\mathrm{d}}-\mathrm{L}_{\mathrm{o}}$ phase separation.

As well as its effect on model membrane systems, high pressure is a fundamental feature of deep-sea environments, ${ }^{14}$ it is employed commercially to inactivate microorganisms ${ }^{15,16}$ there are many examples of locally elevated pressure within cells affecting protein function ${ }^{17}$ and it has important implications in the mechanism of general anaesthesia. ${ }^{18}$ Importantly, lipid bilayers have been shown to be significantly more responsive to hydrostatic pressure than other biomolecules such as proteins or DNA. ${ }^{8,19}$

$\mathrm{L}_{\mathrm{d}}-\mathrm{L}_{\mathrm{o}}$ phase separation has been studied as a function of pressure in multilamellar systems formed from binary ${ }^{20}$ and ternary lipid mixtures, using FT-IR, small angle X-ray diffraction $(\mathrm{SAXS})^{19,21}$ and NMR. ${ }^{22}$ However, all these methods probe the ensemble average structure. Many biological assemblies and their model analogues are highly heterogeneous and so bulk probe technique have significant limitations.

A notable example of the study of the effect of high pressure on individual lipid vesicles is outlined by Nicolini $e t a l .{ }^{23}$ In this study a fused silica capillary pressure cell ${ }^{24}$ is used to visualize morphological changes in the canonical raft mixture of DOPCSM-Chol at constant temperature. Multiphoton fluorescence 
microscopy was used with the fluorescent probe Laurdan to visualize membrane budding under pressure, and the generalized polarization function gives an indication of the packing of the lipids in the membrane, however, membrane domains are not directly observed. It should be noted that the use of a cylindrical capillary optical pressure cell causes significant refraction of the light ${ }^{24}$ and so limits both the field of view and optical resolution of the system.

We have developed a high pressure microscopy cell that allows simultaneous induction and visualization (by wide field fluorescence microscopy) of phase separation in model membranes. The use of flat sapphire optical windows in combination with variable cover slip correction objective lenses means that the images obtained are comparable to those captured in atmospheric pressure microscopy experiments. In addition, because our system is compatible with standard wide field fluorescence microscopy it allows observation of membrane dynamics with sub-second time resolution. We have been able to visualize the temperature and pressure dependent lateral structuring in giant unilamellar vesicles (GUVs), and have been able to follow the dynamic evolution of this structuring in real time (including domain size and morphology).

GUVs composed of 1,2-diphytanoyl-sn-glycero-3-phosphocholine (DPhPC), 1,2-diphytanoyl-sn-glycero-3-phosphocholine, (DPPC) and cholestanol in the ratio $1: 2: 1$ were produced using the electroformation method. ${ }^{25,26}$ At atmospheric pressure, this mixture is known to show phase separation at temperatures below $\sim 42{ }^{\circ} \mathrm{C}^{27}$ and all the components are fully saturated which reduces the possibility of photo-induced oxidation of the lipids.

The fluorescent lipid 1,2-dipalmitoyl-sn-glycero-3-phosphoethanolamine- $N$-(lissamine rhodamine B sulfonyl) (ammonium salt) Rh-DPPE is known to selectively partitions into $\mathrm{L}_{\mathrm{d}}$ domains $^{28}$ and was added at $0.8 \mathrm{~mol} \%$.

The GUVs were contained in a custom built high pressure microscopy cell mounted on a Nikon Eclipse TE2000-E inverted microscope. The cell comprises a high tensile strength stainless steel body with $1 \mathrm{~mm}$ thick, $5 \mathrm{~mm}$ diameter sapphire optical windows, which can withstand pressures of approximately 2500 bar.
Hydrostatic pressure was applied to the sample via a water filled pressure generator (4000 bar, Si-Tec) and hydraulic network similar to that described previously. ${ }^{29}$ Further details can be found in the ESI. $\dagger$

A circulating water bath was connected to the pressure cell to control the temperature, this allows the GUVs to be heated above their mixing transition temperature giving uniform fluid phase vesicles. At constant temperature, the pressure was increased at approximately 4 bar s $^{-1}$ and images were recorded (with temperature and pressure logs) approximately every 0.5 seconds. Pressure propagates extremely fast through the system $^{29}$ and so at this relatively slow pressure ramp rate, the pressure can be considered to be at equilibrium. As the pressure is increased, phase separation is induced as shown by the appearance of dark $\mathrm{L}_{\mathrm{o}}$ areas from the original single bright phase (Fig. 1 and Videos in ESI $\dagger$ ).

Fig. 1 shows the onset of this pressure induced phase separation, and it is clear that there are two distinct mechanisms by which domains form and ripen. Fig. 1A shows a nucleation type mechanism where numerous small round domains are formed, which do not appear to merge or grow with a further increase in pressure. Interestingly, the $\mathrm{L}_{\mathrm{o}}$ domains appear to have a semiordered packing within the bilayer plane which suggests that they are repelling each other, however their circular shape suggests significant line tension between the $\mathrm{L}_{\mathrm{d}}$ and $\mathrm{L}_{\mathrm{o}}$ phases.

The second mechanism as shown in Fig. 1B is a spinodal decomposition type mechanism similar to that shown by Keller et $a l^{6}{ }^{6}$ during temperature induced domain formation. The domains form in labyrinth type structures and the $\mathrm{L}_{\mathrm{o}}$ areas coalesce to become larger with subsequent increases in pressure.

Fig. 1A and B were taken from a single field of view within one sample and during the same pressure ramp, so the experimental conditions for each are identical. The difference in domain formation mechanism between these two vesicles suggests that there is significant inhomogeneity in the composition (and possibly excess membrane area) of vesicles grown by electroformation and it is interesting to note the range of dynamic domain morphology that can occur even in simplified model membranes under the same conditions.
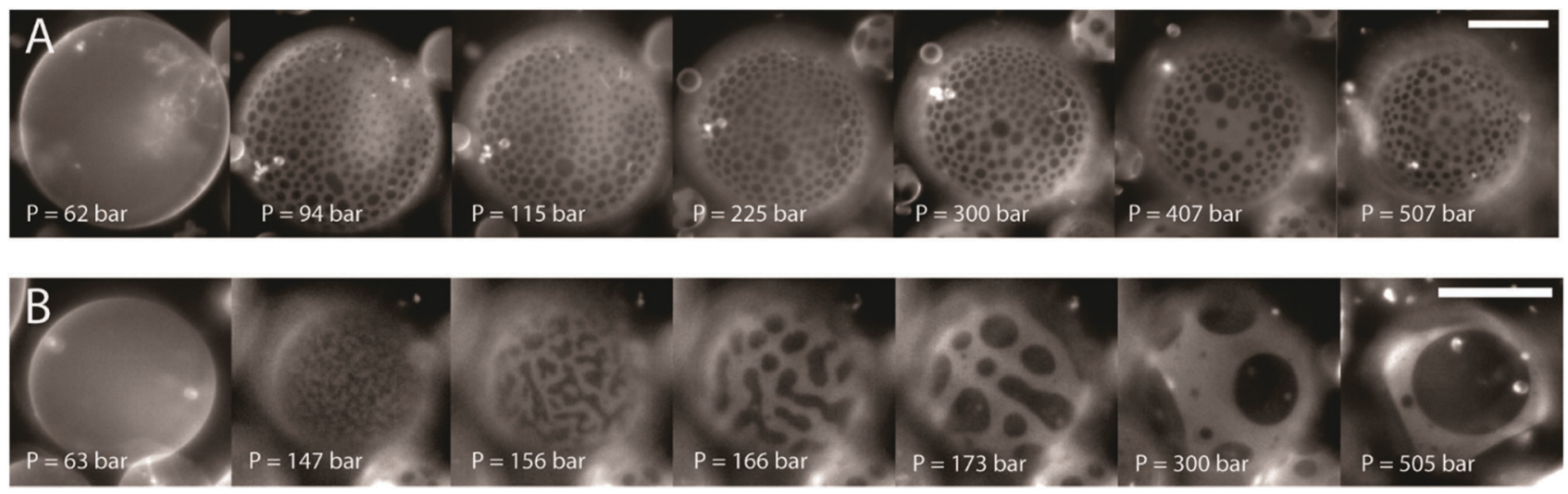

Fig. 1 Pressure induced phase separation of GUVs with the composition 1:2:1 DPhPC, DPPC, cholestanol at $45^{\circ} \mathrm{C}$. (A) shows the induction of many small nucleated $L_{\circ}$ domains and subsequent increases in pressure do not cause domain coalescence, (B) shows a spinodal decomposition type mechanism, where an increase in pressure leads to the merging and growth of $L_{\circ}$ domains. Scale bars are $30 \mu \mathrm{m}$. 


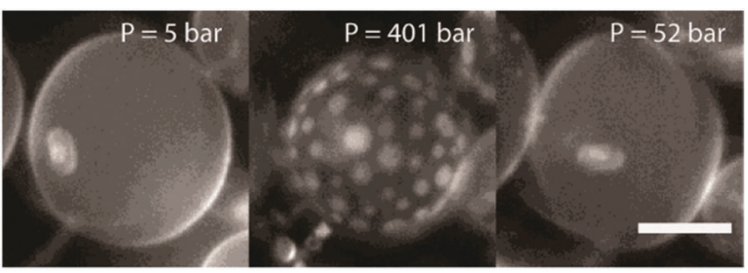

Fig. 2 Pressure induced phase separation of GUVs, with a subsequent decrease in pressure a homogeneous fluid lamellar phase is restored. The reversal of the continuous/discontinuous phases seen here at high pressure compared to Fig. 1 highlights the likely compositional heterogeneity between vesicles. Note, the slight shadowing visible at low pressures is due to the optical properties of the sapphire windows of the pressure cell. Scale bars are $30 \mu \mathrm{m}$.

The induction of domains with pressure is entirely reversible; Fig. 2 shows a vesicle at $42{ }^{\circ} \mathrm{C}$ which forms a homogenous fluid lamellar phase, pressure can be applied to induce separation to coexisting $L_{d}$ and $L_{o}$ phases and when the pressure is released, the vesicle returns to a homogeneous fluid state. This process can be repeated several times to cycle the induction of phase separation and remixing. During repeated pressure cycling, it was noted that the membrane exhibited an increase in excess area and a corresponding reduction in the apparent membrane tension (as shown by an increase in the magnitude of fluctuations). This is thought to occur due to the expulsion of water as the lipid surface area is reduced under pressure, so the internal volume is reduced to minimize the membrane elastic stress. ${ }^{19}$

Although there appear to be two distinct formation mechanisms for the observed membrane domains, the pressure range over which the phase separation onset occurs in individual GUVs at one temperature is narrow. Fig. 3a shows the pressure at which phase separation became apparent in GUVs as a function of temperature. Each point is an average of $10 \mathrm{GUVs}$ in the same sample. There appears to be a linear relationship between temperature and onset pressure within each sample as predicted by the Clapeyron equation. ${ }^{10}$

In addition to fluorescence microscopy, small angle X-ray diffraction (SAXS) was used to probe the induction and pressure response of fluid-fluid phase separation. While the microscopy experiments described above give information about lateral membrane structuring, including domain size and morphology, SAXS probes the bulk structure of lipid mesophase samples at significantly higher resolution and so can resolve the lamellar repeat distance which is related to the bilayer thickness. In addition, high pressure SAXS can be rapidly carried out over a wider temperature and pressure range and since it is a label free technique, it can help exclude the possibility that the addition of small amounts of fluorescently labelled lipid required for fluorescence microscopy alters the phase behaviour of the model system. The long range alignment of domains ${ }^{24}$ allows the detection of phase separation due to the mismatch in thickness of the coexisting liquid phases. Full details of the pressure cell used for X-ray studies have been described previously, ${ }^{29}$ and all SAXS experiments were carried out at beamlines I22, Diamond Light Source (Didcot, UK) and ID02, European Synchrotron Radiation Facility (Grenoble, France).

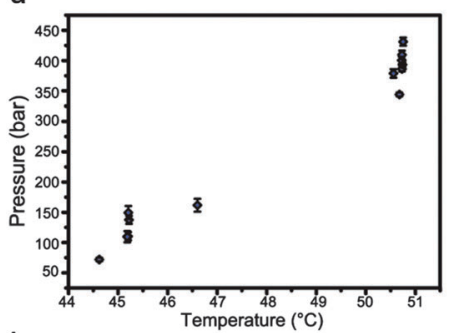

b

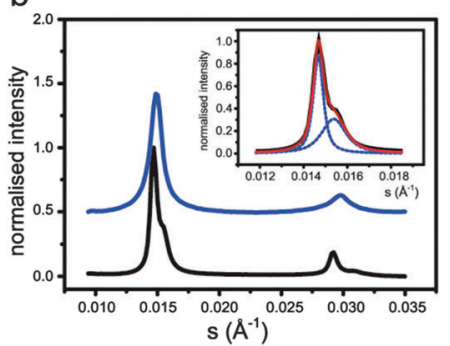

C

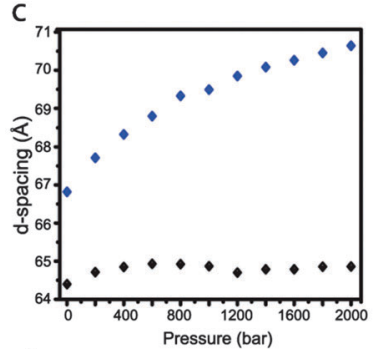

d

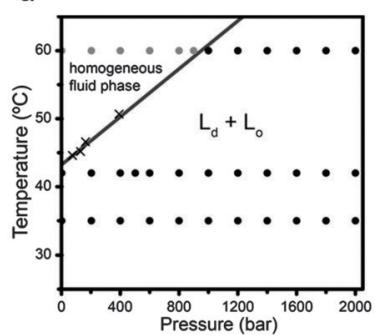

Fig. 3 (a) Phase separation pressure in individual GUVs as a function of temperature. Each point is an average of 10 GUVs in a single sample. (b) Radially integrated small angle X-ray diffraction patterns at $34^{\circ} \mathrm{C}$ (black) and $60^{\circ} \mathrm{C}$ (blue line), both at atmospheric pressure. Inset shows the two Voigt functions (blue lines) fitted to the first order peaks at $34{ }^{\circ} \mathrm{C}$ (experimental data in black) and the sum of the fitted peaks (red line) for comparison with the experimental data. (c) The effect of pressure on the lattice parameters of the two lamellar phases at a constant temperature of $42^{\circ} \mathrm{C}$. (d) Pressure-temperature phase diagram for the same lipid mixture compiled from SAXS and single GUV experiments, crosses mark phase separation pressures measured in GUVs by microscopy, black circles represent SAXS measurements showing $L_{d}-L_{o}$ phase separation and grey circles represent SAXS measurements showing a single homogeneous fluid phase.

Fig. 3b shows integrated diffraction patterns collected at $34{ }^{\circ} \mathrm{C}$ and $60{ }^{\circ} \mathrm{C}$, both at atmospheric pressure. At $60{ }^{\circ} \mathrm{C}$ a homogeneous $L_{d}$ phase is observed showing a characteristic single set of equally spaced diffraction peaks which index to a lamellar phase with a lattice parameter of $68.1 \AA$ A. At $34{ }^{\circ} \mathrm{C}$, which is below the miscibility temperature for this lipid composition, the diffraction pattern shows splitting of the each of the lamellar peaks which can be fitted to two overlapping Voigt functions. The centre of these peaks are used to index the two coexisting lamellar structures which are found to have lattice parameters of $65.8 \AA$ and $69.2 \AA$ at atmospheric pressure. The difference in the lattice parameters between the two phases increases with pressure as shown in Fig. 3c. The height mismatch between the $\mathrm{L}_{\mathrm{d}}$ and $\mathrm{L}_{\mathrm{o}}$ regions is thought to contribute significantly to the line tension at the interface between them ${ }^{30}$ and this appears to correspond to the fluorescence microscopy results in Fig. 1B, which show a reduction in the line interface roughness as the pressure increases (it should be noted that the reduction in the line interface length in Fig. 1B is very likely to be due to domain coarsening. over time).

Fig. 3d shows a pressure-temperature phase diagram for this system constructed using both SAXS data from bulk lipid mesophases and fluorescence microscopy results from GUV experiments. SAXS data was collected from 1-2000 bar at temperatures between 35 and $60{ }^{\circ} \mathrm{C}$. Each 2D SAXS pattern 
was radially integrated and the peaks fitted to single or double Voigt functions as appropriate. Peaks that fitted well to a single Voigt (in which case a double Voigt fit tended to give either two coincident peaks or one with unrealistic parameters) are assigned to a homogeneous fluid lamellar phase, and conditions that required a double Voigt function to fit the peak profile were assigned to coexisting $\mathrm{L}_{\mathrm{d}}$ and $\mathrm{L}_{\mathrm{o}}$ phases. The average GUV phase separation pressure measured in microscopy experiments at a range of temperatures between $40{ }^{\circ} \mathrm{C}$ and $50{ }^{\circ} \mathrm{C}$ correlate very well with the phase separation pressure measured by SAXS at $60{ }^{\circ} \mathrm{C}$ as shown by straight phase boundary that fits to both sets of data.

The rapid propagation and equilibration of pressure allows the structural rearrangements discussed here to be studied in real time on a sub-second time scale which is significantly shorter than has been available previously. It is clear from the results presented here that on initial induction of phase separation in GUVs, the domains are extremely small and highly dynamic. The SAXS experiments also shows that pressure can be used to control the relative thickness of phase separated membrane structures highly precisely and over pressure ranges that are unlikely to significantly perturb other biomolecules.

The technology and methodology that we have developed here offers a valuable insight into the formation of phase separated microdomains in model membranes and we hope that it will catalyse a significantly greater understanding of the link between phase separation in model membrane and dynamic micro domain formation in biological membranes.

We acknowledge Diamond Light Source and the European Synchrotron Radiation Facility for provision of synchrotron beamtime and we would like to thank Dr Andy Smith for assistance using beamline I22 (DLS) and Dr Michael Sztucki for assistance using beamline ID02 (ESRF). This work was supported by EPSRC Platform grant EP/G00465X/1, EPSRC Programme grant EP/J017566/1 and by an EPSRC Centre for Doctoral Training Studentship from the Institute of Chemical Biology EP/F500076/1 awarded to Nicola McCarthy.

\section{Notes and references}

1 D. A. Brown and E. London, Annu. Rev. Cell Dev. Biol., 1998, 14, 111-136.

2 K. Simons and D. Toomre, Nat. Rev. Mol. Cell Biol., 2000, 1, 31-39.

3 C. A. Day and A. K. Kenworthy, Biochim. Biophys. Acta, 2009, 1788, 245-253.

4 S. Munro, Lipid rafts, Cell, 2003, 115, 377-388.

5 S. L. Veatch and S. L. Keller, Phys. Rev. Lett., 2005, 94, 148101.

6 S. L. Veatch and S. L. Keller, Biophys. J., 2003, 85, 3074-3083.

7 M. Edidin, Annu. Rev. Biophys. Biomol. Struct., 2003, 32, 257-283.

8 W. Dzwolak and R. Winter, Philos. Trans. R. Soc. London, Ser. A, 2005, 363, 537-563.

9 R. Winter and C. Jeworrek, Soft Matter, 2009, 5, 3157.

10 N. J. Brooks, O. Ces, R. H. Templer and J. M. Seddon, Chem. Phys. Lipids, 2011, 164, 89-98.

11 A. Squires, R. Templer, J. Seddon, J. Woenkhaus, R. Winter, T. Narayanan and S. Finet, Phys. Rev., 2005, 72, 1-16.

12 A. I. I. Tyler, G. C. Shearman, N. J. Brooks, H. Delacroix, R. V. Law, R. H. Templer, O. Ces and J. M. Seddon, Phys. Chem. Chem. Phys., 2011, 13, 3033-3038.

13 C. Conn, O. Ces, X. Mulet, S. Finet, R. Winter, J. Seddon and R. Templer, Phys. Rev. Lett., 2006, 96, 1-4.

14 A. Picard and I. Daniel, Biophys. Chem., 2013, 183, 30-41.

15 D. Knorr, V. Heinz and R. Buckow, Biochim. Biophys. Acta, 2006, 1764, 619-631.

16 N. Rivalain, J. Roquain and G. Demazeau, Biotechnol. Adv., 2010, 28, 659-672.

17 D. Marsh, Biochim. Biophys. Acta, 1996, 1286, 183-223.

18 P. L. Chau, Br. J. Pharmacol., 2010, 161, 288-307.

19 C. Nicolini, J. Kraineva, M. Khurana, N. Periasamy, S. S. Funari and R. Winter, Biochim. Biophys. Acta, 2006, 1758, 248-258.

20 M. Pühse, C. Jeworrek and R. Winter, Chem. Phys. Lipids, 2008, 152, 57-63.

21 C. Jeworrek, M. Pühse and R. Winter, Langmuir, 2008, 24, 11851-11859.

22 R. Winter, Annu. Rep. NMR Spectrosc., 2003, 50, 163-200.

23 C. Nicolini, A. Celli, E. Gratton and R. Winter, Biophys. J., 2006, 91, 2936-2942.

24 J. D. Müller and E. Gratton, Biophys. J., 2003, 85, 2711-2719.

25 M. I. Angelova and D. S. Dimitrov, Faraday Discuss. Chem. Soc., 1986, 303-311.

26 M. I. Angelova, S. Soléau, P. Méléard, F. Faucon and P. Bothorel, Preparation of giant vesicles by external AC electric fields. Kinetics and applications, Progress in Colloid and Polymer Science, Steinkopff, 1992, vol. 89, pp. 127-131.

27 S. L. Veatch, K. Gawrisch and S. L. Keller, Biophys. J., 2006, 90, 4428-4436.

28 T. Baumgart, G. Hunt, E. R. Farkas, W. W. Webb and G. W. Feigenson, Biochim. Biophys. Acta, 2007, 1768, 2182-2194.

29 N. J. Brooks, B. L. Gauthe, N. J. Terrill, S. E. Rogers, R. H. Templer, O. Ces and J. M. Seddon, Rev. Sci. Instrum., 2010, 81, 064103.

30 F. A. Heberle, R. S. Petruzielo, J. Pan, P. Drazba, N. Kučerka, R. F. Standaert, G. W. Feigenson and J. Katsaras, J. Am. Chem. Soc., 2013, 135, 6853-6859. 